\title{
Das türkische Verfassungsgericht auf dem Weg zum „Hüter der Verfassung“"
}

\author{
Julia Platter und Barı̧̧ Çalı̧̧kan*
}

Am 27. April 2007 stellte sich Abdullah Gül, bis dato Außenminister der Türkei, als Kandidat der $\mathrm{AKP}^{1}$, einer islamisch-konservativ ausgerichteten Partei, die auch die Regierung stellte, in der Großen Türkischen Nationalversammlung (GNVT) zur Wahl des Staatspräsidenten. Er scheiterte im ersten und auch im zweiten Wahlgang an der erforderlichen Zweidrittelmehrheit. Die Oppositionsfraktion der CHP hatte die Wahl, wie von ihr bereits zuvor angekündigt, boykottiert. Am 1. Mai 2007 erklärte der türkische Verfassungsgerichtshof - Anayasa Mahkemesi - den ersten Wahlgang für die Wahl des Präsidenten der Republik auf Antrag der CHP-Fraktion für ungültig. Die Nationalversammlung beschloss daraufhin vorgezogene Neuwahlen. Die Entscheidung des Verfassungsgerichtshofs war in der Türkei sehr umstritten und rief auch im Ausland ein starkes Medienecho hervor. Sie ist umso beachtenswerter, als das Gericht schon circa ein Jahr später wiederum zum „Schiedsrichter" in der politischen Auseinandersetzung zwischen dem religiös-konservativen und dem national-kemalistischen Lager im Parteiverbotsverfahren gegen die Regierungspartei AKP des amtierenden Ministerpräsidenten Recep Tayyip Erdoğan aufgerufen war (Urteilsverkündung am 30. Juli 2008). ${ }^{2}$ Während jedoch das (gegen die AKP gescheiterte) Parteiverbotsverfahren - aus deutscher Sicht ungewohnt - zum „klassischen Arsenal“ und zu den oft verhandelten Verfahren vor dem türkischen Verfassungsgericht gehört, beschreitet die Entscheidung zur Präsidentenwahl verfahrensrechtlich neue Wege. Ungeachtet der politischen Bedeutung des Parteiverbotsverfahrens zu Lasten der AKP ist die Entscheidung zur Präsidentenwahl vor allen Dingen interessant, weil hier das türkische Verfassungsgericht seine Entscheidungsbefugnisse im Zusammenspiel mit dem Parlament indirekt neu definiert.

\section{Die Verfassungsgerichtsbarkeit in der modernen Türkei}

Bereits die erste Verfassung des türkischen Nationalstaats von 1924, der das Osmanische Reich nach dem Ersten Weltkrieg ablöste, orientierte sich am Prinzip der Gewaltenteilung

* Zurzeit der Entstehung des Beitrags war Barış Çalı̧̧kan Lehrbeauftragter an der rechtswissenschaftlichen Fakultät der Universität Hamburg.

1 In der laufenden - 23. - Wahlperiode sind neben der AKP (Adalet ve Kalkinma Partisi / Partei der Gerechtigkeit und des Wiederaufbaus), die CHP (Cumhuriyet Halk Partisi / Republikanische Volkspartei), die MHP (Millliyetçi Hareket Partisi / Partei Nationaler Bewegung), die DTP (Demokratik Toplum Partisi / Partei demokratischer Gesellschaft) und die DSP (Demokratik Sol Partisi / Linkspartei) in der Großen Nationalversammlung der Türkei vertreten. In der 22. Wahlperiode waren neben der AKP und der CHP unter anderem Kandidaten der ANAP (Anavatan Parti / Heimatspartei) und der DYP (Doğru Yol Parti / Partei des Rechten Weges) vertreten; nicht aber MHP, DTP und DSP.

2 Ausführlich zu politischen Hintergründen dieser Entscheidung Dirk Tröndle, Das Verbotsverfahren gegen die Partei für Gerechtigkeit und Entwicklung (AKP), die Türkische Regierungspartei, KAS Auslandsinformationen, 8-9/08, S. 68 - 96, die Entscheidungsgründe können eingesehen werden unter http://www.anayasa.gov.tr/general (Abruf am 15. November 2008). 
mit der Trennung von Legislative, Exekutive und Judikative. Einen eigenständigen Verfassungsgerichtshof sah indes diese Verfassung noch nicht vor. Ob stattdessen den Fachgerichten die Kompetenz zur Normenkontrolle einzuräumen sei, war in der türkischen verfassungsrechtlichen Diskussion seinerzeit umstritten. ${ }^{3}$ Ohnehin war aber das Rechtsstaatsprinzip insgesamt in jener Verfassung noch unvollkommen entwickelt. ${ }^{4}$ Die Verfassung konnte nicht verhindern, dass die Republik Türkei sich Ende der fünfziger Jahre hin zur Diktatur einer Partei entwickelte. ${ }^{5}$ Diese Entwicklung beendete das Militär, das sich bis heute als Hüter der Prinzipien des Staatsgründers der modernen Türkei Kemal Atatürk versteht, im Jahre 1960 durch einen Putsch und initiierte eine Verfassungsreform. Die daraus entstandene Verfassung von 1961 konnte zum damaligen Zeitpunkt als sehr liberal gelten. ${ }^{6}$ Das darin als Verfassungsorgan konzipierte erste türkische Verfassungsgericht nahm seine Tätigkeit am 22. April 1962 auf der Grundlage eines Verfassungsgerichtsgesetzes auf.7

Der Verfassung von 1961 war jedoch keine lange Geltung beschieden. Starke politische Spannungen veranlassten das Militär im Jahre 1971 erneut zum Eingreifen und zu einer Verfassungsrevision, in der einige der liberalen Errungenschaften von 1961 zurückgenommen wurden. ${ }^{8}$ Dennoch beruhigte sich in den folgenden Jahren die politische Lage nicht. 1980 wurde nach einem erneuten Putsch eine Militärdiktatur errichtet, und zwei Jahre später, am 7. November 1982, eine von den Militärs vorgelegte neue Verfassung durch einen Volksentscheid angenommen. ${ }^{9}$ Damit entfernte man sich noch weiter von bereits erreichten liberalen Standards mit der Begründung, dies sei „zur Wahrung der Einheit und Souveränität des Landes“ erforderlich. ${ }^{10}$ Die Einrichtung des Verfassungsgerichts wurde jedoch beibehalten.

In den Jahren 2001 und 2004 wurde die Verfassung, die bis dahin noch deutlich den Stempel ihrer oktroyierten Herkunft trug, mit Blick auf einen möglichen Beitritt der Türkei zur Europäischen Union und die dafür bestehenden Anforderungen an Grundrechtsstandards und Demokratisierung reformiert. ${ }^{11}$ Insbesondere wurden die Befugnisse des

3 Darstellung bei Mustafa Yildiz, Die Verfassungsgerichtsbarkeit in der Türkei - Gerichtliche Nachprüfung der Verfassungsmäßigkeit der Gesetze, Diss. Göttingen 1979, S. 27 ff.

4 Zusammenfassung der Defizite bei Yavuz Abadan, Die Türkische Verfassung von 1961, in: JöR 13 (1964), S. 326, S. 329.

5 Ebenda, S. 326, S. 330 ff.; Ernst E. Hirsch, Die Verfassung der Türkischen Republik vom 9. November 1982, in: JöR 32 (1983), S. 507, S. 510; Christian Rumpf, Das türkische Verfassungssystem, Wiesbaden 1996, S. 64; Ergun Özbudun, in: Don Wallace, Jr. (Hrsg.), Anayasa Introduction in Turkish Law, Den Haag 2007, S. 22.

6 Ernst E. Hirsch, a.a.O. (Fn. 5), S. 507, S. 509.

7 Osman Can, Die Entwicklung der türkischen Verfassungsgerichtsbarkeit, http://www.anayasa.gov. tr/eskisite/verfass.pdf (Abruf am 1. September 2007).

8 Ernst E. Hirsch, a.a.O. (Fn. 5), S. 507, S. $511 \mathrm{f}$.

9 Dazu Wulf Damkowski, Die neue türkische Verfassung - „Fahrplan zur Demokratie“?, in: EuGRZ 1984, S. 1 (S. 1).

10 Ebenda mit Hinweisen auf die vom Parlamentarischen Rat des Europarates in Auftrag gegebenen Gutachten, Fn. 4 und passim; Ernst E. Hirsch, a.a.O. (Fn. 5), S. 512, Christian Rumpf, in: KlausDetlev Grothusen (Hrsg.), Südosteuropa-Handbuch Türkei, Göttingen 1985, S. 117.

11 Udo Steinbach, Die Türkei und die EU, in: APuZ, B 33 / 34 (2004), S. 3 ff.; Jeanne Lätt / Asiye Öztürk, Zypern und die türkisch-europäischen Beziehungen, in: APuZ, B 43 (2007), S. 33, S. 37. So bezeichnet Mehmet A. Irtemçelik, Die Europäische Union und die Türkei: Fehler vermeiden, in: RuP 40 (2004), S. 78, S. 80, die Anerkennung des Beitrittskandidatenstatus als ,ver- 
nationalen Sicherheitsrates eingeschränkt und damit die verfassungsrechtlich abgesicherte Einflussnahme des Militärs auf das Staatsleben zurückgedrängt. Die Bürgerrechte wurden verfassungsrechtlich gestärkt. ${ }^{12}$

\section{Das Verfassungssystem der Türkei heute}

Die zentrale Vorschrift der türkischen Verfassung (TV) von 1982 ist Art. 2. Diese Bestimmung benennt die Staatsgrundsätze. Verglichen mit Art. 20 GG legt sie nicht nur die Verfassungsprinzipien der Rechts- und Sozialstaatlichkeit und das Bekenntnis zur Demokratie nieder, sondern auch den Nationalismus Atatürks (Kemalismus) und den Laizismus. Anders als die Bundesrepublik ist die Türkei ein Zentralstaat. ${ }^{13}$

Der Text der Verfassung zeichnet sich durch eine - jedenfalls im Gegensatz zum Grundgesetz - sehr ausführliche und auf Vollständigkeit bedachte Formulierung seiner 177 Artikel aus. In ihrer Struktur entspricht sie mit einem Grundrechtsteil und einem anschließenden staatsorganisatorischen Teil dem Muster vieler europäischer Verfassungen. ${ }^{14}$

\subsection{Entscheidung für eine starke Stellung des Staatspräsidenten}

Ein wesentlicher Unterschied zwischen dem Staatsaufbau nach dem Grundgesetz und der türkischen Verfassung liegt in der verfassungsrechtlich starken Stellung des Staatspräsidenten (Art. 104 TV). Er wird gemäß Art. 102 TV von der Großen Türkischen Nationalversammlung (GNVT) auf sieben Jahre gewählt. Seine Befugnisse und Aufgaben beschränken sich, anders als die des Bundespräsidenten ${ }^{15}$, nicht auf Repräsentationsaufgaben. Zwar werden die eigentlichen Regierungsgeschäfte vom Ministerrat wahrgenommen, der Staatspräsident hat jedoch viele Möglichkeiten der Einflussnahme. Er ernennt und entlässt den Mi-

diente Antwort“ auf die Reformen in der Türkei. Deutsche Übersetzung der Verfassung der türkischen Republik von 1982 in der aktuellen Fassung unter http://www.verfassungen.de/tr/tuerkei82-index.htm.

12 Günter Seufert, Türkei, in: Werner Weidenfeld / Wolfgang Wessels (Hrsg.), Jahrbuch der Europäischen Integration 2001 / 2002, Bonn 2002, S. 441 ff. Einen Überblick über diese Verfassungsreformen gibt Heinz Kramer, Die Türkei im Prozess der Europäisierung, in: APuZ, B 33 / 34 (2004), S. 9 ff. Siehe zu den Grundrechten insbesondere Gesetz Nr. 4709 vom 3. Oktober 2001.

13 Zum Exekutivaufbau siehe Christian Rumpf, a.a.O. (Fn. 10), S. 169, 194 ff.

14 Eine solche Struktur findet sich außerhalb des Grundgesetzes der Bundesrepublik Deutschland insbesondere in den Niederlanden und Spanien. Das erste Kapitel der niederländischen Verfassung („Grondwet voor het Koninkrijk der Nederlanden“) beginnt mit einem grundrechtlichen Teil („Grondrechte“). Die folgenden Kapitel widmen sich der Staatsorganisation. Eine ebensolche Parallele findet sich in der Verfassung von Spanien. Im ersten Abschnitt der spanischen Verfassung, der sich den Staatsprinzipien anschließt, werden die Grundrechte („De los derechos y deberes fundamentales“) festgeschrieben. Dem folgt der staatsorganisationsrechtliche Aufbau („El sistema de Gobierno"). Im zweiten und dritten Abschnitt statuiert die spanische Verfassung das Königreich („De la Corona“) und die Volksvertretung („De la Cortes Generales“).

15 Ingolf Pernice, in: Horst Dreier (Hrsg.), Grundgesetzkommentar, Band 2, Art. 20-82, 2. Auflage, Tübingen 2006, Art. 54 Rn. 14 - 16; Michael Nierhaus, in: Michael Sachs (Hrsg.), Grundgesetzkommentar, 4. Auflage, München 2007, Art. 54 Rn. 6 f. 
nisterpräsidenten und seine Minister, darf selbst den Ministerrat einberufen, kann dem Volk Referenden für Verfassungsänderungen unterbreiten, entscheidet über den Einsatz der türkischen Streitkräfte, sitzt dem so genannten Staatskontrollrat mit dessen umfangreichen Untersuchungs- und Kontrollkompetenzen in allen Bereichen der Verwaltung vor (Art. 108 TV) und ernennt einen Teil der Richter der Obergerichte (Art. 146). Sehr umfassend sind seine Befugnisse im Zusammenhang mit dem staatlichen Notstand (Art. 119 bis 122 TV). ${ }^{16}$ Des Weiteren beruft er die Mitglieder des Verfassungsgerichts (Art. 104 Abs. 2 c und 146 TV ${ }^{17}$ und ist oberster Hüter der Verfassung (Art. 104 Abs. 1, 2. Halbs. TV). Diese grobe Aufstellung zeigt bereits, dass der Staatspräsident eine Schlüsselposition einnimmt. So verwundert es nicht, dass die Wahl des Staatspräsidenten in einer veränderten politischen Landschaft Gegenstand intensiver, auch staatsrechtlicher Auseinandersetzungen ist.

\subsection{Grundprinzipien und Ewigkeitsklausel}

Ähnlich wie das Grundgesetz in Art. 79 Abs. 3 mit den dort genannten Prinzipien und dem darin enthaltenen Verweis auf Art. 1 und 20 nehmen in der türkischen Verfassung die in Art. 1 (Staatsform Republik) und 2 TV und der Präambel (Prinzipien des Staatsgründers Mustafa Kemal Atatürk, so genannter Kemalismus) ${ }^{18}$ genannten Prinzipien einen besonderen Rang ein: Sie können nicht abgeändert werden (Art. 4 TV). Einzelne Grundrechte sind als solches nicht Bestandteil der Ewigkeitsklausel. In ihren Schutz einbezogen ist allgemein nur der „die Menschenrechte achtende Rechtsstaat“. Insofern sind die Grundrechte und Grundfreiheiten der türkischen Verfassung grundsätzlich nicht abänderungsfeindlich. Doch hat das Verfassungsgericht in mehreren Entscheidungen klargestellt, dass die Grenzen der zulässigen Änderungen im Rahmen des Prinzips der Rechtsstaatlichkeit erreicht wären, wenn Grundrechte und Grundfreiheiten vollständig abgeschafft würden. ${ }^{19}$ Darüber hinausgehend interpretiert das Verfassungsgericht den Inhalt des Rechtsstaatsprinzips insoweit auch positiv, als es klarstellt, dass „ein Rechtsstaat derjenige Staat ist [...], der die Menschenrechte achtet [und] diese Freiheiten schützt und stärkt ${ }^{\text {“20 }}$. Damit ist der grundrechtliche Schutz nicht der Disposition des Gesetzgebers überlassen, sondern findet seinen Halt in eben jenem Verfassungsprinzip der Rechtsstaatlichkeit.

16 Die Stellung des Staatspräsidenten und seine Aufgaben erinnern damit von fern an diejenigen des Reichspräsidenten nach der Weimarer Reichsverfassung (der allerdings direkt vom Volk gewählt wurde, Art. 41 WRV); siehe zu dessen angenommener Stellung als „Wahrer und Hüter des verfassungsmäßigen Zustandes und des verfassungsmäßigen Funktionierens der obersten Reichsinstanzen" und zugleich zur Entstehung dieses Begriffs, Carl Schmitt, Der Hüter der Verfassung, in: AöR 16 n.F. (1929), S. 161 ff.

17 Die Ernennungsbefugnis des Präsidenten ist jedoch insoweit eingeschränkt, als er nur drei der elf Verfassungsrichter aus originärer Entscheidungsmacht ernennen darf. Nach Art. 146 Abs. 2 TV ist die Kandidatenaufstellung respektive das „bindende Vorschlagsrecht“ der obersten Gerichtsbarkeit zugewiesen.

18 Zum ideengeschichtlichen Hintergrund und zu der (partei-)politischen Entwicklung dieses Begriffes siehe weiterführend Bekim Agai, Islam und Kemalismus in der Türkei, in: APuZ, B 33 / 34 (2004), einsehbar unter http://www.das-parlament.de/2004/33-34/beilage/004.html (Abruf am 1. September 2007).

19 TürkVerfG, AMKD (Anayasa Mahkemesi Kavarlar Dergesi = Entscheidungssammlung des Verfassungsgerichts) 4, S. 290 (S. 329).

20 TürkVerfG, AMKD 21, S. 35 (S. 59); 22, S. 323 (S. 365); 23, S. 47 (S. 71). 


\section{Das Verfassungsgericht der Türkei heute}

Die türkische Verfassung behandelt das Verfassungsgericht als einen Teil der „Rechtsprechung "im dritten Abschnitt des zweiten Hauptteils im Kapitel II zu den obersten Gerichten (Art. 148 bis 153 TV). Dort wird es an erster Stelle genannt, aber nicht als eigenständiges Verfassungsorgan ausdrücklich hervorgehoben. ${ }^{21}$ Dennoch kann man aufgrund der Aufgaben und Kompetenzen auf eine sachlich herausragende und von den übrigen hohen Gerichten zu unterscheidende Stellung schließen. ${ }^{22}$ Sowohl die Funktionen als auch die des Gerichts sind bereits in der Verfassung enthalten und nicht etwa dem Gesetzgeber überlassen (Art. 146 TV). ${ }^{23}$ Als einziges Gericht kann es Gesetze für nichtig erklären (Art. 153 Abs. 3 TV). Seine Entscheidungen binden alle übrigen Verfassungsorgane sowie alle natürlichen und juristischen Personen (Art. 153 Abs. 6 TV). ${ }^{24}$

\subsection{Verfahrensarten}

Aus Tabelle 1 geht hervor, dass die türkische Verfassungsgerichtsbarkeit sich am Vorbild europäischer Schwesterinstitutionen orientiert. Auf diese Weise unterstreicht das türkische Verfassungsrecht seine Zugehörigkeit zum Kreis europäischer Verfassungen.

\begin{tabular}{|c|c|}
\hline Bundesverfassungsgericht & Anayasa mahkemesi \\
\hline $\begin{array}{l}\text { abstrakte Normenkontrolle, } \\
\text { Art. } 93 \text { Abs. } 1 \text { Nr. 2, } 2 \text { lit. a) GG }\end{array}$ & Anfechtungsklage, Art. 150 TV \\
\hline konkrete Normenkontrolle, Art. 100 GG & Vorlageverfahren, Art. $152 \mathrm{TV}$ \\
\hline $\begin{array}{l}\text { Organstreitverfahren, } \\
\text { Art. } 93 \text { Abs. } 1 \text { Nr. } 1 \text { GG }\end{array}$ & $\begin{array}{l}\text { kein Gegenstand des Verfahrens vor dem Verfassungs- } \\
\text { gericht mit der Ausnahme des Abgeordnetenschutzver- } \\
\text { fahrens in Art. } 85 \text { TV (Anfechtungsverlangen gegen } \\
\text { den Mandatsverlust oder- Aufhebungsbeschluss der } \\
\text { Immunität und Indemnität) }\end{array}$ \\
\hline $\begin{array}{l}\text { Individualverfassungsbeschwerde, } \\
\text { Art. } 93 \text { Abs. } 1 \text { Nr. } 4 \text { lit. a) GG }\end{array}$ & $\begin{array}{l}\text { kein Gegenstand des Verfahrens vor dem Verfassungs- } \\
\text { gericht }\end{array}$ \\
\hline $\begin{array}{l}\text { Zuständigkeit in übrigen im Grundgesetz } \\
\text { vorgesehenen Fällen, } \\
\text { Art. } 93 \text { Abs. } 1 \text { Nr. } 5 \text { GG: } \\
\text { - Parteiverbot, Art. } 21 \text { Abs. } 2 \text { GG }\end{array}$ & $\begin{array}{l}\text { Zuständigkeit in übrigen in der türkischen Verfassung } \\
\text { vorgesehenen Fällen, } \\
\text { Art. } 148 \text { Abs. } 7 \text { TV: } \\
\text { - Parteiverbot, Art. } 69 \text { Abs. } 4 \text { TV }\end{array}$ \\
\hline Quelle: Eigene Zusammenstellung. & \\
\hline
\end{tabular}

21 Wie übrigens auch das Bundesverfassungsgericht nicht, siehe insofern den IX. Abschnitt des GG „Rechtsprechung“, dort Art. 94 GG.

22 Vgl. zum Organstreitverfahren vor dem Bundesverfassungsgericht Christian Pestalozza, Verfassungsprozessrecht, 3. Auflage, München 1991, \$2 Rn. 13.

23 Siehe aber das Gesetz zum Aufbau und der Gerichtsbarkeit des Verfassungsgerichts (Anayasa Mahkemesi Kuruluş ve Görevleri Hakkinda Nr. 2949).

24 Vgl. auch die Übersicht über Mitglieder, Aufgaben und Tätigkeit des Verfassungsgerichts in deutscher Sprache, hrsg. vom Verfassungsgericht der Türkei, http://www.anayasa.gov.tr/images/loaded/pdf_dosyalari/anayasa_album_alm_opt.pdf (Abruf am 1. September 2007). 
Ähnlich wie im Grundgesetz sind die vor dem türkischen Verfassungsgericht zulässigen Verfahren enumerativ geregelt, das heißt, eine allgemeine „Zuständigkeit für verfassungsrechtliche Streitigkeiten" gibt es nicht. ${ }^{25}$ Eine verfassungsgerichtliche Kontrolle staatlicher Maßnahmen ist nur dort eröffnet, wo eine ausdrückliche Ermächtigung durch die Verfassung besteht. ${ }^{26}$

Die beiden wichtigsten Verfahrensarten, die dem türkischen Verfassungsgericht zugewiesen sind, sind die so genannte Anfechtungsklage, eine Form der abstrakten Normenkontrolle (Art. 150 TV), und das Vorlageverfahren (Art. 152 TV) als konkrete Normenkontrolle. Daneben entscheidet das Verfassungsgericht auch über das Verbot politischer Parteien oder sonstige Einschränkungen für die Tätigkeit einer Partei (Art. 69 Abs. 3 und 8 TV). Eine Entsprechung zum Organstreitverfahren, das im Rahmen der Aufgabenzuweisungen an Verfassungsgerichte in Deutschland die rechtshistorisch älteste Aufgabenzuweisung darstellt $^{27}$, kennt die türkische Verfassung nicht. Aus bundesdeutscher Perspektive „fehl “ " auch die Verfassungsbeschwerde, die dem einzelnen Bürger in Deutschland unmittelbaren verfahrensrechtlichen Zugang zum Verfassungsgericht verschafft. ${ }^{28}$

\subsection{Die Anfechtungsklage gemäß Art. 150 TV (abstrakte Normenkontrolle): das türkische Verfassungsgericht als Verfassungshüter „light“?}

Die Anfechtungsklage gemäß Art. 150 TV ist, ähnlich dem grundgesetzlichen Pendant der abstrakten Normenkontrolle, als objektives Beanstandungsverfahren ausgestaltet. Eines konkreten Anlasses oder eines konkreten Streits über die Verfassungsmäßigkeit einer Norm bedarf es nicht. Antragsbefugt sind der Präsident der Republik, die Fraktionen der Regierungspartei und der größten Oppositionspartei sowie ein Fünftel der Mitglieder der Nationalversammlung. Prüfungsgegenstand in diesem Verfahren kann sein (1) ein Parlamentsgesetz, (2) eine Rechtsverordnung mit Gesetzeskraft und (3) die Geschäftsordnung des Parlaments (GO-GNVT). Damit ist die Zahl der möglichen Verfahrensgegenstände im isolierten Vergleich zur abstrakten Normenkontrolle vor dem Bundesverfassungsgericht ge-

25 Vgl. dazu auch die Übersicht bei Osman Can, a.a.O. (Fn. 7).

26 TürkVerfG, AMKD 4, S. 204 (S. 206); 8, S. 433 (S. 448); 26, S. 391 (S. 402); Ernst E. Hirsch, Die Verfassung der Türkischen Republik, Staatsverfassungen der Welt (Bd. 7), Frankfurt am Main 1966, Art. 147, Erl. Nr. 2, S. 181.

27 Dieter Umbach, in: ders. / Thomas Clemens / Franz-Wilhelm Dollinger, BVerfGG - Mitarbeiterkommentar, 2. Auflage, Heidelberg 2005, Vorb. \$\$ 63 ff. Rn. 7; Christian Pestalozza, a.a.O. (Fn. 22), $\$ 7$ Rn. 7; Jost Pietzcker, Organstreit, in: Peter Badura / Horst Dreier (Hrsg.), Festschrift 50 Jahre Bundesverfassungsgericht, Bd. 1, Tübingen 2001, S. 587, S. 588.

28 Das führt zu einer signifikant hohen Zahl von Individualbeschwerden gemäß Art. 35 EMRK vor dem Europäischen Gerichtshof für Menschenrechte, dazu Nilay Aras, Die Bedeutung der EMRK für den Grundrechtsschutz in der Türkei, in: ZEuS 2007, S. 219 (S. 238). Ob nicht auch die türkische Verfassung der Einrichtung der Verfassungsbeschwerde bedarf, wird dennoch gefragt, vgl. die Diskussion während des Symposiums zum 42-jährigen Bestehen des türkischen Verfassungsgerichtshofes im April 2004, an dem der damalige Vizepräsident des Bundesverfassungsgerichts Winfried Hassemer teilgenommen hat. Vgl. hierzu nur die lebendig geführte Diskussion über die Zweckmäßigkeit der Einführung einer Verfassungsbeschwerde in: Anayasa Yargisi (Jahrbuch zur Verfassung) 21, S. $204-229$. 
mäß Art. 93 Abs. 1 Nr. 1 GG, \$\$ 13 Nr. 6, 76 ff. BVerfGG etwas erweitert ${ }^{29}$, der Prüfungsgegenstand unterliegt allerdings einer speziellen Begrenzung: Eine Besonderheit des türkischen Verfassungsrechts besteht darin, dass der Gerichtshof verfassungsändernde Gesetze nur daraufhin prüfen darf, ob sie unter Beachtung des vorgeschriebenen Verfahrens und in der gehörigen Form zustande gekommen sind (Art. 148 Abs. 1 S. 2 und Abs. 2 S. 1 TV). Mag daher auch die türkische Verfassung mit einer „Ewigkeitsklausel“ versehen sein, ist es jedenfalls nach dem Wortlaut der Verfassung nicht das Verfassungsgericht, das für die Bewahrung der dort genannten Prinzipien in letzter Instanz verantwortlich scheint. Vielmehr ist dies, wie oben bereits angedeutet, eine Aufgabe des Staatspräsidenten (Art. 104 Abs. 1 S. 2, 2. HS TV). Dieser hat beispielsweise die Möglichkeit, verfassungsändernde Gesetze dem Volk zur Abstimmung zu unterbreiten (Art. 104 a Abs. 5 TV) oder ein Gesetz an die Türkische Nationalversammlung zur erneuten Verhandlung zurückzuleiten (Art. 104a Abs. 4 TV).

Mithin lässt sich feststellen, dass die Stellung des türkischen Verfassungsgerichts nicht ohne weiteres mit der des Bundesverfassungsgerichts zu vergleichen ist; seine Aufgaben und Kompetenzen sind enger gesteckt. Deshalb kann das türkische Verfassungsgericht nicht in gleicher Weise, wie es das Bundesverfassungsgericht für sich in Anspruch nehmen kann, als „Hüter der Verfassung “30 bezeichnet werden. Ohne eine materielle verfassungsgerichtliche Überprüfung von Verfassungsänderungen erfüllt insbesondere die „Ewigkeitsklausel“ Art. 4 TV zunächst nur eine „Appellfunktion“, da das Verfassungsgericht als unabhängiges Organ über ihre Beachtung nicht wachen kann.

\section{Erweiterung des Prüfungsmaßstabs}

Der Verfassungsgerichtshof hat durchaus die ihm als Verfassungsorgan zustehende Kompetenz-Kompetenz für sich in Anspruch genommen und seine Prüfungskompetenzen im Laufe der Zeit weit ausgelegt.

In der Verfassung von 1961 war weder positiv noch negativ geregelt, ob das Gericht auch Verfassungsänderungen am Maßstab der bereits in dieser Verfassung enthaltenen Ewigkeitsklausel überprüfen konnte. Das Verfassungsgericht ging indes davon aus und stellte dafür die Gesetzesqualität der Verfassungsänderung in den Vordergrund. ${ }^{31}$ Zur Überprüfung von Gesetzen war das Verfassungsgericht nämlich bereits entsprechend dem Wortlaut der Verfassung ermächtigt (Art. 147 TV [1961]). ${ }^{32}$

29 Wobei zu bedenken ist, dass Bestimmungen der Geschäftsordnung des Bundestags im Rahmen des Organstreitverfahrens gemäß Art. 93 Abs. 1 Nr. 1 GG, $\$ \$ 13$ Nr. 5, 63 ff. BVerfGG durchaus Gegenstand der verfassungsgerichtlichen Prüfung sein können, vgl. Thomas I. Schmidt, Die Geschäftsordnung der Verfassungsorgane als individuell-abstrakte Regelungen des Innenrechts, in: AöR 28 (2003), S. 608, S. 644 ff; Jost Pietzcker, Schichten des Parlamentsrechts: Verfassung, Gesetze und Geschäftsordnung, in: Hans-Peter Schneider / Wolfgang Zeh, Parlamentsrecht und Parlamentspraxis in der Bundesrepublik Deutschland, Berlin / New York 1989, \$10 Rn. 44 ff.

30 Reinhold Zippelius / Thomas Würtenberger, Deutsches Staatsrecht, 31. Auflage, München 2005, $\$ 48$, S. 442; kritisch hierzu unter Herausarbeitung der Grenzen verfassungsgerichtlicher Kompetenzen Klaus J. Grigoleit, Bundesverfassungsgericht und deutsche Frage, Tübingen 2004, \$ 3 III, S. $87-106$.

31 Yavuz Abadan, a.a.O. (Fn. 4), S. 336, S. 340 f., S. 382.

32 Türk. VerfGE, AMKD 8, S. 313 (S. 322 f.). 
Der Verfassungsgeber versuchte jedoch schon wenige Jahre später, die Zuständigkeiten des Gerichts wieder zu beschränken. Mit der Reform von 1971 wurde hinsichtlich verfassungsändernder Gesetze festgelegt, dass die Kontrollbefugnis des Verfassungsgerichtshofs sich lediglich auf die formelle Verfassungsmäßigkeit erstrecken sollte (Art. 147 TV [1971]). In mehreren aufeinander aufbauenden Entscheidungen interpretierte das Gericht seine Prüfungskompetenz jedoch in der Zusammenschau mit weiteren Verfassungsbestimmungen. Als zentrale Vorschriften betrachtete es hierbei Art. 4 und 148 TV. Seit 1971 stellt Art. 4 TV die Staatsform der Türkei als Republik - Cumhuriyet - unter besonderen Schutz und entzieht die Wahl der Staatsform dem parlamentarischen Gesetzgeber. Das Verfassungsgericht folgerte daraus, dass in die Nationalversammlung keine Gesetzentwürfe eingebracht werden können, die Änderungen hinsichtlich der republikanischen Staatsform enthalten, da dem Parlament hierfür die Zuständigkeit fehle, solche also bereits formell verfassungswidrig seien. ${ }^{33}$ Dies dürfe vom Verfassungsgerichtshof weiterhin überprüft werden. Da zu den Inhalten des Republikprinzips auch der Grundsatz der Rechtsstaatlichkeit gehört (Art. 4 in Verbindung mit Art. 2 TV), sieht sich das Verfassungsgericht befugt, verfassungsändernde Gesetze auch am Maßstab des Rechtsstaatsprinzips, letztlich also inhaltlich zu prüfen. ${ }^{34}$ Folglich ist jedenfalls dort, wo der Gesetzentwurf mit der republikanischen Staatsform in Berührung kommt und insoweit „republikwidrig“ sein kann, der Weg zur verfassungsgerichtlichen Kontrolle von Verfassungsänderungen eröffnet.

Die vom Verfassungsgericht entwickelte Interpretation seiner Prüfungskompetenzen erinnert entfernt an die „klassische“ Diskussion der Prüfungskompetenzen des deutschen Bundespräsidenten bei der Ausfertigung von Gesetzen. Obwohl der Wortlaut des Grundgesetzes eher für ein bloß formelles Prüfungsrecht des Bundespräsidenten zu sprechen scheint, ist in der Staatspraxis seit langem akzeptiert, dass dieser im Falle eines evidenten Verfassungsverstoßes die Ausfertigung von Gesetzen verweigern kann. ${ }^{35}$ In diesem Zusammenhang wird überwiegend angenommen, dass die Prüfung der verfassungsmäßigen Form eines Gesetzes mit Blick auf die besonderen Formanforderungen für das verfassungsändernde Gesetz zugleich die inhaltliche Prüfung verlangt, ob ein Gesetz die Verfassung durchbricht und damit ändert, eine eindeutige Trennung einer formalen gegenüber einer inhaltlichen

33 Türk. VerfGE, AMKD 13, S. 403 (S. 430); 14, S. 118 (S. 131); in der Entscheidung Türk. VerfGE, AMKD 14, S. 118 (S. 131) führt das Verfassungsgericht aus, dass ein Gesetz, welches das Ziel der Abänderung der in Art. 2, 4 TV genannten Staatsformmerkmale verfolge, weder eingebracht noch angenommen werden kann (,... bu ilkeleri değiştirmeyi amac güden herhangi bir kanun teklif ve kabul edilemez").

34 Türk. VerfGE, AMKD 13, S. 403 (S. 430 f.).

35 Zur Staatspraxis vgl. Hartmut Maurer, in: Rudolf Dolzer / Klaus Vogel / Karin Graßhof (Hrsg.), Bonner Kommentar zum Grundgesetz, 54. Lieferung, Art. 82 (1988) Rn. 31 - 33. Das BVerfG hat zur Prüfungskompetenz des Bundespräsidenten noch nicht eingehend Stellung genommen, aber mehrfach - in obiter dicta - eine solche angenommen, siehe zum Beispiel BVerfGE 1, S. 396 (S. 413 f.); 2, S. 143 (S. 169); 34, S. 9 (S. 22 f.); dies wird auch von der überwiegenden Mehrheit in der Literatur vertreten, siehe zum Beispiel Hartmut Bauer, in: Horst Dreier (Hrsg.), a.a.O. (Fn. 15), Art. 82 Rn. 13; Bodo Pieroth, in: Hans D. Jarass / ders. (Hrsg.), Grundgesetz für die Bundesrepublik Deutschland: Kommentar, 7. Auflage, München 2004, Art. 82 Rn. 3; Hartmut Maurer, a.a.O. (Fn. 35), Art. 82 Rn. 44; Reinhold Zippelius / Thomas Würtenberger, a.a.O. (Fn. 30), \$ 45, S. $402 \mathrm{f}$. 
Prüfung also nicht möglich ist. ${ }^{36} \mathrm{Im}$ Gegensatz zu den knappen Formulierungen und dem weitreichend auslegungsfähigen Text des Grundgesetzes waren die interpretatorischen Hürden, die das türkische Verfassungsgericht zu nehmen hatte, sehr viel höher. Immerhin schreibt Art. 148 Abs. 1 S. 2 TV (1982) nicht nur vor, dass verfassungsändernde Gesetze lediglich formell überprüft werden dürfen; Art. 148 Abs. 2 S. 2 TV (1982) definiert vielmehr auch ausdrücklich, was unter diese formelle Prüfung fällt, nämlich nur die Frage, ob der Mehrheit für Vorschlag und Abstimmung sowie der Bedingung, dass nicht im Eilverfahren verhandelt wurde, entsprochen wurde.

Das Verfassungsgericht hält sich jedoch mit der - wenn auch angreifbaren - Begründung seiner Prüfungskompetenz an anerkannte Auslegungsmethoden. Der Verknüpfung von Art. 2 und 4 TV liegt eine systematische Betrachtung zu Grunde. Das Gericht räumt damit demjenigen Gesichtspunkt bei der Lösung verfassungsrechtlicher Fragestellungen den Vorzug ein, der einheits- und bestandserhaltend in Bezug auf die grundlegenden Verfassungsprinzipien wirkt.

\section{Einbeziehung weiterer Prüfungsgegenstände: die Präsidentenwahl vom 27. April 2007}

Ein besonderer Anlass für das türkische Verfassungsgericht, seine Zuständigkeiten (erweiternd) zu definieren, war die Präsidentenwahl in der GNVT vom 27. April 2007. Obwohl die Verfassung ihrem Wortlaut nach kein Organstreitverfahren, sondern im Grundsatz nur verschieden ausgeformte Normenkontrollverfahren vorsieht, erklärte das Verfassungsgericht die Wahl für verfassungswidrig.

Da die siebenjährige Amtszeit des bisherigen Amtsinhabers Ahmet Necdet Sezer am 16. Mai 2007 endete, war das Parlament aufgerufen, einen neuen Präsidenten zu wählen (Art. 101, 102 TV). Verfassungsgemäß besteht die Nationalversammlung aus 550 Mitgliedern (Art. 75 TV). Da im ersten oder zweiten Wahlgang nur zum Staatspräsidenten gewählt ist, wer zwei Drittel der Stimmen der Mitglieder des Parlaments auf sich vereinigen kann (Art. 102 Abs. 1 TV), benötigt ein Kandidat 367 Stimmen, um gewählt zu werden. Im dritten Wahlgang genügt die absolute Mehrheit, also 276 Stimmen (Art. 102 Abs. 3 TV). Dem von der Regierungspartei AKP mit 352 Sitzen im Parlament vorgeschlagenen Abdullah Gül wurde vor Beginn der Wahl von seinen politischen Gegnern, unter anderem der CHP, vorgeworfen, den in der Verfassung niedergelegten Grundsatz des Laizismus aushebeln zu wollen. In der Folge boykottierten die in der Opposition befindlichen Fraktionen der CHP, der ANAP und der DYP den ersten Wahlgang in der GNVT, indem ihre Mitglieder der Abstimmung fernblieben. ${ }^{37}$ Gül erhielt daraufhin erwartungsgemäß im ersten Wahlgang nicht die erforderliche Zweidrittelmehrheit, überdies hatten an der Abstimmung aufgrund des Boykotts weniger als zwei Drittel der Mitglieder des Parlaments teilgenommen. Die Oppo-

36 Beispielsweise Theodor Maunz, in: ders. / Günter Dürig (Begr.), Grundgesetz, 1. Lieferung, Art. 82 (1958) Rn. 2; kritisch hierzu zum Beispiel Brun-Otto Bryde, in: Ingo von Münch / Philip Kunig (Hrsg.), Grundgesetz-Kommentar, Bd. III, 5. Auflage, München 2003, Art. 82 Rn. 6.

37 Die von der CHP schon vorab angekündigte parlamentarische Taktik wurde bereits vorher verfassungsrechtlich diskutiert, vgl. dazu Rainer Hermann, Präsidentenwahl nach Boykott in der Schwebe, Bericht vom 27. April 2007, http://www.faz.net/s/RubDDBDABB9457A437BAA85A49C26FB23A0/Doc-EAE66FAF05B2A479ABE6AA3BB4D09934B $\sim A T p l-E c o m$ mon-Scontent.html (Abruf am 9. Oktober 2007). 
si-tionsfraktion CHP stellte im Folgenden fristgerecht einen Antrag gemäß Art. 150 TV (Anfechtungsklage) vor dem Verfassungsgericht, um den Wahlgang annullieren zu lassen. Ihrer Auffassung nach hätten für einen verfassungsgemäßen Ablauf der Wahl bereits während des ersten Wahlgangs mindestens zwei Drittel aller Abgeordneten anwesend sein müssen. Das Verfassungsgericht gab dem Antrag statt. ${ }^{38}$

\subsection{Parlamentarische Beschlüsse als zulässige Prüfungsgegenstände gemäß Art. 150 TV?}

Auf den ersten Blick scheint es verblüffend, dass das Verfassungsgericht sich in der Lage sah, über diesen Parlamentsakt zu entscheiden, da, wie schon erläutert, das Organstreitverfahren im Katalog der Zuständigkeiten des Verfassungsgerichts nicht vorhanden ist, sondern nur das Normenkontrollverfahren gemäß Art. 150 TV zur Verfügung steht. Danach kann Prüfungsgegenstand (Art. 150 S. 1 TV) neben Gesetzen und Verordnungen mit Gesetzeskraft auch eine Bestimmung der GO-GNVT sein. Eine Prüfungsbefugnis zu (Einzel-) Beschlüssen des Parlaments kann dem Wortlaut der Verfassung demgegenüber nicht entnommen werden. ${ }^{39}$ Das Verfassungsgericht selbst hat in seiner bisherigen Rechtsprechung immer betont, dass eine verfassungsgerichtliche Kontrolle nur dort eröffnet ist, wo in dieser Hinsicht eine ausdrückliche Ermächtigung in der Verfassung besteht. ${ }^{40}$ Eine „Generalklausel“, die es grundsätzlich gestatten würde, das Verfassungsgericht in allen verfassungsrechtlichen Streitigkeiten anzurufen, gibt es - wie bereits erwähnt - nicht. ${ }^{41}$ Eine gerichtliche Praxis, nach der auch (Einzel-)Beschlüsse des Parlaments ohne weiteres als Bestimmungen der Geschäftsordnung anzusehen wären, existiert ebenso wenig. ${ }^{42}$

Nach dem bisher Gesagten stand der Wortlaut des Art. 150 TV einer verfassungsgerichtlichen Kontrolle des Wahlgangs am 27. April 2007 entgegen. Das Verfassungsgericht war daher unter anderem vor die Aufgabe gestellt, überhaupt seine Zuständigkeit zur Entscheidung über diese Frage zu begründen, also einen im Rahmen des Verfahrens nach Art. 150 ff. TV zulässigen Prüfungsgegenstand ausfindig zu machen. In diesem Zusammenhang waren für das Gericht die Identifizierung des Prüfungsgegenstandes und die materiell-rechtliche Begründung der Entscheidung aufs Engste verknüpft.

Nach Auffassung des Gerichts verstieß die Feststellung des Parlamentspräsidenten zu Beginn der Sitzung, in der der erste Wahlgang durchgeführt werden sollte, das Parlament sei gemäß Art. 96 TV beschlussfähig und damit auch abstimmungsfähig im Rahmen des Wahlverfahrens zum Staatspräsidenten, gegen die speziellere Abstimmungsfähigkeitsregel des Art. 102 TV. Diese mache neben der qualifizierten Mehrheit (zwei Drittel) für den Wahlerfolg des Kandidaten auch eine Anwesenheit von mindestens zwei Dritteln aller gewählten Abgeordneten erforderlich. ${ }^{43}$

38 TürkVerfG, Urteil vom 1. Mai 2007, E.S. 2007/45, KS 2007/54.

39 Anders Mustafa Yildiz, a.a.O. (Fußn. 3), S. $121 \mathrm{f}$.

40 TürkVerfG, AMKD 4, S. 204 (S. 206); 8, S. 433 (S. 448); siehe auch Ernst E. Hirsch, a.a.O. (Fn. 26), Art. 147, Erläut. Nr. 2, S. 181.

41 Dazu TürkVerfG, AMKD 26, S. 391 (S. 402); zuletzt Urteil des türkischen VerfG vom 1. Mai 2007, a.a.O. (Fn. 38), S. 9.

42 Verfassungsgesetzlich geregelte Ausnahme ist die Anfechtungsklage gegen den Beschluss über Aufhebung der Immunität eines Abgeordneten gemäß Art. 85 TV.

43 Urteil des türkischen VerfG vom 1. Mai 2007, a.a.O. (Fn. 38), S. 15. 
Zunächst erscheint es überflüssig, neben dem in Art. 102 Abs. 3 TV ausdrücklich vorgesehenen Abstimmungsquorum von zwei Dritteln der Mitglieder ein Anwesenheitsquorum in gleicher Höhe für alle Wahlgänge - also auch bereits für den ersten - zu postulieren: Schließlich ist die Anwesenheit dieser qualifizierten Mehrheit von vornherein erforderlich, damit im ersten oder zweiten Wahlgang überhaupt ein Kandidat erfolgreich gewählt werden kann. Allerdings war sowohl der Regierungsfraktion der AKP, der Oppositionspartei der CHP als Antragstellerin als auch dem Verfassungsgericht klar, dass es auf den dritten Wahlgang ankommen würde, bei dem gemäß des in Art. 102 Abs. 3 TV vorgesehenen Abstimmungsquorums die absolute Mehrheit für einen erfolgreichen Wahlgang genügen würde. ${ }^{44}$ Dies geht aus der Antragsbegründung der CHP-Fraktion hervor, in der darauf hingewiesen wird, dass das für die „Staatspräsidentenwahl erforderliche Anwesenheitsquorum nicht nur im ersten, sondern in allen Wahlgängen zu suchen sei“ ${ }^{45}$. Beim damaligen Kräfteverhältnis zwischen der AKP und der Opposition hätte dies bedeutet, dass die Wahl eines Staatspräsidenten aus der Regierungsfraktion für die Dauer der Legislaturperiode faktisch ausgeschlossen sein würde, forderte man auch im dritten Wahlgang die Anwesenheit von zwei Dritteln der gesetzlichen Mitglieder der GNVT, denn über so viele Mandate verfügte die AKP-Fraktion nicht. Erst aus dieser Perspektive wird im Übrigen auch die BoykottAktion der Oppositionsfraktionen überhaupt erst verständlich. ${ }^{46}$

Das Verfassungsgericht machte sich die Auffassung der Antragstellerin im Grundsatz zu eigen und stellte in Bezug auf Art. 102 Abs. 1 TV fest, dass dieser eine Sondervorschrift zu Art. 96 TV (Beschlussfähigkeit) für die Wahl des Präsidenten enthalte. ${ }^{47}$ Auch wenn es in der Entscheidung nur sehr zurückhaltend zum Ausdruck kommt, hatte auch das Gericht - ebenso wie die politischen Akteure - vor allen Dingen also den dritten Wahlgang im Blick.

Bevor allerdings das Verfassungsgericht sich der Frage widmen konnte, ob die Verfassung während des gesamten Wahlvorgangs ein Anwesenheitsquorum vorschreibt, musste es zunächst einen geeigneten Prüfungsgegenstand finden. Dieses Problem der Zulässigkeit war der eigentlichen Auslegung von Art. 102 Abs. 1 und 3 TV vorgelagert, hing aber im konkreten Fall untrennbar damit zusammen. Prüfungsgegenstand war hier nach Auffassung des Verfassungsgerichts die dem Wahlvorgang vorausgegangene Feststellung des Parlamentspräsidenten, dass das Parlament gemäß Art. 96 Abs. 1 TV beschlussfähig sei. Diesen Beschluss ordnete das Gericht als Geschäftsordnungsregelung und damit zulässigen Prüfungsgegenstand im Sinne des Art. 148 TV ein. Nach seiner Auffassung wurde mit diesem Beschluss nämlich nicht allein eine - möglicherweise - verfassungswidrige Feststellung über die Beschlussfähigkeit des Parlaments getroffen. Eine solche Einzelfallentscheidung hätte, trotz des Umstandes, dass sie möglicherweise gegen die Verfassung verstieß, nicht Prüfungsgegenstand eines Normenkontrollverfahrens gemäß Art. 150 TV sein können. Vielmehr ord-

44 Dies geht gerade aus der Antragsbegründung hervor, in der darauf hingewiesen wird, dass die Anwesenheitsmehrheit nicht nur für den ersten Wahlgang gelte, sondern auch für die übrigen einzuhalten sei $(, \ldots$ toplanti yeter sayinin Cumurbaşkani seçiminde yalniz birinci oylamada değil fakat dört oylamanin her birisinde de aranmasi gerektiği, 102 nci maddenin kaleme aliniş biçiminden açikca anlaşilmaktadir"), Urteil des türkischen VerfG vom 1. Mai 2007, a.a.O. (Fn. 38), S. 3 .

45 Ebenda.

46 Dazu Rainer Hermann, a.a.O. (Fn. 37).

47 Urteil des türkischen VerfG vom 1. Mai 2007, a.a.O. (Fn. 38), S. 12. 
nete das Verfassungsgericht dieser Entscheidung die Wirkung eines GeschäftsordnungsÄnderungsbeschlusses $\mathrm{zu}^{48}$, da sie auch den normativen, geschäftsordnungs-ändernden Inhalt habe, dass das in $\$ 121$ GO-GNVT in Verbindung mit Art. 102 Abs. 1 TV enthaltene Anwesenheitsquorum bei der Wahl des Staatspräsidenten nicht beachtet zu werden brauchte. ${ }^{49} \$ 121$ GO-GNVT sieht vor, dass die Präsidentenwahl entsprechend Art. 102 TV erfolgt. Der Beschluss war also nach Auffassung des Verfassungsgerichts insofern doppelfunktional, als er einen konkret-individuellen feststellenden und einen abstrakt-generellen verfahrensbezogenen Anteil hatte. Dass der Parlamentsbeschluss nicht explizit als Änderungs- oder Abweichungsbeschluss gefasst worden war, steht der Annahme einer Geschäftsordnungsänderung nicht entgegen. ${ }^{50}$ Von der Warte des Verfassungsgerichtshofes war es hierbei im Weiteren unerheblich, ob die Geschäftsordnung selbst Abweichungsbeschlüsse im Einzelfall gestattet beziehungsweise hierfür besondere Bedingungen vorschreibt. ${ }^{51}$ So oder so gilt, dass Verfahrensbestimmungen der Geschäftsordnung nicht gegen die ihnen übergeordneten verfassungsrechtlichen Vorgaben verstoßen dürfen.

Zum besseren Verständnis sei noch erwähnt, dass der Parlamentspräsident der GNVT, ungleich dem Präsidenten des Deutschen Bundestages, nach türkischem Parlamentsrecht eine ausdrückliche Feststellung über die Beschlussfähigkeit des Hauses treffen muss. Anders als im Bundestag, dessen Beschlussfähigkeit im Grundgesetz nicht geregelt ist ${ }^{52}$, verlangt die türkische Verfassung ein Anwesenheitsquorum von einem Drittel der Mitglieder, „soweit die Verfassung nichts Anderes [also Strengeres] bestimmt" (Art. 96 Abs. 1 S. 1 TV). Die Feststellung der Beschlussfähigkeit ist somit konstitutiv für die formelle Verfassungsmäßigkeit der nachfolgenden Parlamentsakte.

\subsection{Die qualifizierte Anwesenheitsmehrheit als Inhalt von Art. 102 TV}

Nachdem das Verfassungsgericht den Prüfungsgegenstand mit der These der Doppelfunktionalität fixiert hatte, konnte es sich der Begründung seiner Auffassung zuwenden, nach der Art. 102 TV neben einer qualifizierten Abstimmungsmehrheit auch ein qualifiziertes Anwesenheitsquorum enthält. Einen systematischen Einstieg lieferte ihm der Wortlaut des

48 Ebenda, S. $14 \mathrm{f}$.

49 Die Unterscheidung in einerseits eine Abweichung von den Regeln der GO und andererseits deren Abänderung hat für die Frage der Prüfungskompetenz keine Bedeutung, da auch die Abweichung von der GO deren Regelungscharakter betrifft und eben für den Einzelfall eine andere Geschäftsordnungsvorschrift gilt, nämlich die durch die Abweichung bestimmte Verfahrensweise, vgl. Jost Pietzcker, a.a.O. (Fn. 29), \$10 Rn. 19.

50 Verfahrensbeschlüsse über die parlamentarische Arbeitsweise liegen auch dann vor, wenn sie nicht als solche veröffentlicht und bezeichnet sind, ebenda, $\$ 10$ Rn. 19.

51 Siehe hierzu $\$ 126$ GO-BT, der Abweichungsbeschlüsse ausdrücklich gestattet, aber hierfür eine Zwei-Drittel-Mehrheit der anwesenden Mitglieder des Bundestages verlangt und (selbstverständlich) bestimmt, dass ein solcher Beschluss nicht gegen die Vorschriften des Grundgesetzes verstoßen darf.

52 Dazu BVerfG, Beschluss vom 10. Mai 1977, 2 BvR 705/75, BVerfGE 44, S. 308, worin die von der Geschäftsordnung des Bundestages aufgestellte Vermutung, das Beschlussfähigkeit bestehe, solange nicht ein Antrag auf Feststellung der Beschlussfähigkeit gestellt wird ( $\$ 45$ Abs. 2 GOBT), für verfassungskonform erklärt wird; vgl. auch die ausdrücklich in der Verfassung niedergelegte Vermutungsregel in Art. 33 Abs. 2 BaWüVerf. 
Art. 96 Abs. 1 TV (Beschlussfähigkeit im Allgemeinen), demgemäß diese Bestimmung nur gelten soll, „soweit die Verfassung keine andere Vorschrift enthält“. Das Gericht begründet sodann das qualifizierte Anwesenheitsquorum für die Wahl des Präsidenten gemäß Art. 102 TV mit weiteren systematischen und teleologischen Argumenten.

Zunächst wird die Wahl des Staatspräsidenten mit jener für das Präsidium der Nationalversammlung gemäß Art. 94 Abs. 3 TV verglichen. ${ }^{53}$ Auch diese ist in vier mögliche, aufeinanderfolgende Wahlgänge mit absteigenden Mehrheiten unterteilt, wobei in den ersten beiden eine Mehrheit von zwei Dritteln, im dritten Wahlgang aber nur noch die absolute Mehrheit erforderlich ist.

Den Schwerpunkt seiner Argumentation legt der Gerichtshof auf die teleologische Interpretation des Art. 102 TV und arbeitet dabei zunächst die herausgehobene Stellung des Staatspräsidenten im Verfassungsgefüge heraus. ${ }^{54}$ Diese sei, so wird betont, angesichts der Statuswirkungen des Staatspräsidentenamtes bei der Auslegung der Verfassungsnormen, die sein Amt betreffen, besonders zu berücksichtigen. ${ }^{55}$ Aufbauend auf diesem Argument schließt das Gericht auf das Erfordernis der Anwesenheit von mindestens zwei Dritteln der Mitglieder des Parlaments während des Wahlvorgangs, da nur so eine breite demokratische Legitimation für den zukünftigen Amtsinhaber sichergestellt sei und zwar auch dann, wenn er nicht bereits im ersten oder zweiten Wahlvorgang gewählt werde. ${ }^{56}$ Letztere Aussage kann der Entscheidung nur aus einem als obiter dictum formulierten Halbsatz entnommen werden. ${ }^{57}$ Jedoch liegt in dieser Aussage das eigentliche „Gelenkstück“ der Argumentation des Gerichts, denn, wie erwähnt, fallen das postulierte Anwesenheitsquorum und die erforderliche Stimmenmehrheit erst für den dritten Wahlgang zahlenmäßig auseinander.

Zwangsläufig ist die über den Wortlaut hinausgehende Deutung des Art. 102 Abs. 1 und 3 TV angreifbar. Sie ist auch in der Öffentlichkeit angesichts der seinerzeit spannungsgeladenen politischen Situation heftig kritisiert worden. ${ }^{58}$ So ist es durchaus nicht zwingend, der einleitenden Feststellung des Parlamentspräsidenten über die Beschlussfähigkeit des Parlaments die Bedeutung eines Änderungsbeschlusses zu $\$ 121$ GO-GNVT zuzuweisen. Der Interpretation des $\$ 121$ GO-GNVT in Verbindung mit Art. 102 TV in der Weise, dass dort neben dem Abstimmungsquorum auch ein spezielles Anwesenheitsquorum für die Präsidentenwahl enthalten ist, muss man ebenfalls nicht folgen. In diesem Falle hat die Feststellung des Parlamentspräsidenten über die Beschlussfähigkeit auch keinen geschäftsordnungsändernden Inhalt. Selbst wenn man sich der Auffassung des Verfassungsgerichts anschließt, dass die Wahl des Präsidenten die Anwesenheit von mindestens zwei Dritteln

53 Urteil des türkischen VerfG vom 1. Mai 2007, a.a.O. (Fn. 38), S. 13.

54 Ebenda.

55 Ebenda.

56 Ebenda. Die Frage nach Anwesenheitsquoren ist dem deutschen Verfassungsrecht nicht grundsätzlich fremd. So hatte auch das Bundesverfassungsgericht darüber zu entscheiden, ob aus dem Demokratieprinzip des Art. 20 Abs. 2 GG für die Beschlussfähigkeit des Bundestages ein Anwesenheitsquorum zu fordern sei, BVerfGE 44, S. 308 (S. 315 ff.).

57 Urteil des türkischen VerfG vom 1. Mai 2007, a.a.O. (Fn. 38), S. 12; so führt das Verfassungsgericht aus, dass Art. 102 Abs. 3 TV im Sinne des Art. 96 Abs. 1 TV eine ,andere Vorschrift“ für die Beschlussfähigkeit und Abstimmungsmehrheit ist („üçuncü fikra hükmünün de karar yetersayisi bakimindan, 96. maddedeki toplanti ve karar yetersayisina ilişkin genel kuralin istisnalarini oluşturan, ,Anayasadaki başkaca hükümler" kapsaminda bulunduğu sonucuna ulasilmaktadir.").

58 Vgl. den Bericht von Rainer Hermann, der Professor Ergun Özbudun (Bilkent-Universität Ankara) als verfassungsrechtliche Autorität zitiert, a.a.O. (Fn. 37). 
der Abgeordneten erfordert, kann man an den Zulässigkeitsüberlegungen des Gerichts Kritik üben. Die allgemeine Feststellung der Beschlussfähigkeit des Parlaments könnte auch als unabhängig von einer - hier möglicherweise fehlenden, aber zusätzlich notwendigen - Feststellung über die besonderen Anwesenheitsvoraussetzungen für die Präsidentenwahl betrachtet werden. Auch dann hätte sie keinen geschäftsordnungsändernden Inhalt gehabt, so dass es jedenfalls an einem zulässigen Prüfungsgegenstand (Geschäftsordnungsbestimmung) für ein Verfahren nach Art. 150 TV gefehlt hätte.

Diese gewichtigen Gegenargumente stellen aber die Legitimität der tatsächlich vom Verfassungsgericht getroffenen Entscheidung und ihrer Begründung nicht in Frage. Die gewählte Argumentationsfigur, von der besonderen inhaltlichen Bedeutung eines Rechtsakts auf gesteigerte Verfahrensanforderungen zurückzuschließen, findet beispielsweise auch in der deutschen Verfassungsrechtsprechung zum Staatsorganisationsrecht Entsprechungen. Erinnert sei an die damals ebenfalls umstrittene AWACS-Entscheidung aus dem Jahre 1994, in der das Bundesverfassungsgericht postuliert hatte, dass die Bundeswehr sich an bewaffneten Einsätzen im Ausland („out of area“) im Rahmen eines kollektiven Sicherheitssystems nur mit Zustimmung des Parlaments beteiligen dürfe, obwohl ein solcher parlamentarischer Zustimmungsvorbehalt im Wortlaut des Grundgesetzes nicht ausdrücklich geregelt ist. ${ }^{59}$ Seinerzeit erklärte das Bundesverfassungsgericht den Parlamentsvorbehalt mit historischen und systematischen Argumenten zum Konstruktionsprinzip der Wehrverfassung im Grundgesetz. ${ }^{60}$

Vielleicht noch interessanter im Hinblick auf die aktuelle Entscheidung des türkischen Verfassungsgerichts ist ein Urteil des Bayrischen Verfassungsgerichtshofs aus dem Jahre 1999. ${ }^{61}$ Darin wurde der Erfolg eines Volksentscheids bei Verfassungsänderungen im Wege der Volksgesetzgebung von einer Beteiligung von mindestens 25 Prozent der Stimmberechtigten abhängig gemacht. Nicht nur, dass die Verfassung ein solches Beteiligungsquorum nicht enthielt - der Verfassungsgerichtshof erklärte sogar ausdrücklich eine entgegengerichtete Entscheidung hierzu aus dem Jahre 1949 für überholt. Er begründete dies mit dem notwendigen erhöhten Bestandsschutz der Verfassung und der Gefahr, dass die Anforderungen an eine hinreichende demokratische Legitimation bei zu kleinen Beteiligungsquoren unterschritten würden. Das Gericht betonte, dass die Verfassung hier eine planwidrige Lücke enthalte, die von ihm durch entsprechende rechtsschöpfende Auslegung zu schließen sei.

59 BVerfG, Urteil vom 12. Juli 1994, BvE 3/92, 5/93, 7/93, 8/93, E 90, S. 286 (S. 382 - 388).

60 Die AWACS-Entscheidung weist noch einen weiteren interessanten Berührungspunkt mit der hier besprochenen Entscheidung auf: Die antragstellende FDP-Fraktion wandte sich mit ihrem Antrag gegen Out-of-area-Einsätze, stimmte aber mit ihrer Koalitionspartnerin im Bundestag dafür. Aus diesem Grund verneinten die Richter Ernst-Wolfgang Böckenförde und Konrad Kruis in einem Minderheitsvotum von vornherein die Zulässigkeit des Antrags, BVerfGE 90, S. 286 (S. 389 - 394). Im Fall „Präsidentschaftwahl Gül" prangerte die Antragstellerin, die CHP-Fraktion, letztendlich eine Abstimmungssituation an, die sie selbst absichtlich durch ihren Wahlboykott herbeiführt hatte. Indes sah das türkische Verfassungsgericht keinen Anlass, auf diese Umstände einzugehen. Argumentativ ließe sich die Zurückhaltung respektive das Schweigen des Gerichts damit stützen, dass es sich bei der Anfechtungsklage nach Art. 150 TV eben um ein objektives Beanstandungsverfahren handelt. Sieht man es so, besteht keine prozessuale Möglichkeit, dieses „doppelte Spiel“ bei der Entscheidungsfindung zu berücksichtigen.

61 BayVerfGHE 52, S. 104 (Abschaffung des Bayrischen Senats durch Volksentscheid) = NVwZ-RR 2000, S. 65. 
Die beiden beispielhaft herausgegriffenen Entscheidungen deutscher Verfassungsgerichte sind thematisch insofern vergleichbar, als auch dort hinreichende demokratische Legitimation als Begründung für erhöhte, mehr oder weniger rechtsschöpfend aufgestellte Verfahrensanforderungen herangezogen wurde. Der Vergleich zeigt jedenfalls, dass das türkische Verfassungsgericht sich ungeachtet der politischen Brisanz der Situation durchaus auch anderswo verwendeter verfassungsrechtlicher Argumentationsmuster bedient hat.

\subsection{Die abstrakte Normenkontrolle als „kleines Organstreitverfahren“?}

$\mathrm{Ob}$ die weitreichende Auslegung des Prüfungsgegenstands nur der besonderen Entscheidungssituation geschuldet war, oder ob das Verfassungsgericht seine extensive Interpretation fortentwickeln wird, kann nicht vorhergesagt werden. Immerhin wäre es denkbar, Parlamentsbeschlüsse unter dem in dem hier besprochenen Urteil entwickelten Gesichtspunkt ihrer möglichen Doppelfunktionalität nunmehr allgemein nicht mehr als Prüfungsgegenstand von vornherein auszuschließen. Das könnte beispielsweise der jeweiligen Opposition im Parlament ermöglichen, Mehrheitsentscheidungen außerhalb des Gesetzgebungsverfahrens verfassungsgerichtlich überprüfen zu lassen. Solche prozessualen Möglichkeiten gehen über ein abstraktes Normenkontrollverfahren hinaus und nähern sich dem Verfahrenstyp, der im Verfassungsprozessrecht der Bundesrepublik als Organstreitverfahren bezeichnet wird.

\section{Das Verfassungsgericht im demokratischen Gefüge der türkischen Verfassung}

Im Verhältnis zwischen Bundestag und Bundesverfassungsgericht ist der Grundsatz des judicial self-restraint prägend. Dies bedeutet keine Verkürzung der Zuständigkeiten des Gerichts, sondern den Verzicht darauf, „Politik zu betreiben“62. Die richterliche Selbstbeschränkung stärkt das Gewaltenteilungsprinzip des Grundgesetzes und setzt dem Eingreifen in den von der Verfassung geschaffenen und eingehegten Raum freier politischer Gestaltung des demokratisch gewählten Parlaments Grenzen. Das türkische Verfassungsgericht stand zunächst vor einem anderen Problem, nämlich der Festigung seiner Stellung im Verfassungsgefüge des Landes. Die Verfassung von 1961 wollte mit der Einführung einer Verfassungsgerichtsbarkeit die Willkürherrschaft einer ursprünglich demokratisch gewählten Parlamentsmehrheit beenden, was jene Mehrheiten dennoch nicht davon abhielt, die Zuständigkeiten eines „,unerwünschten“ Gerichts zu beschneiden und seine Bedeutung zu relativieren. Seit Inkrafttreten der Verfassung von 1982 wiederum sah das Gericht gegenüber einem restriktiven Verfassungssystem seine Aufgabe darin, das Rechtsstaatsprinzip zu stärken und hatte in dieser Lage wenig Anlass, sich mit Fragen des judicial self-restraint auseinanderzusetzen. Vielmehr musste es seine politische Rolle gegenüber dem Gesetzgeber erst etablieren. ${ }^{63}$ Daher ist es verfehlt, dem Gericht anlässlich der Entscheidung zur Wahl des Staatspräsidenten eine unzulässige politische Einmischung vorzuwerfen.

62 BVerfGE 36, S. 1 (S. 14 f.); kritisch zur Beachtung dieses Grundsatzes durch das Gericht Minderheitenvotum BVerfGE 93, S. 121 (S. 151 f.).

63 Christian Rumpf, Das türkische Verfassungsgericht und die Grundzüge seiner Rechtsprechung, in: EuGRZ 1990, S. 129, S. 135 mit weiteren Nachweisen. 
Auch die Umstände des Parteiverbotsverfahrens gegen die AKP verweisen nicht auf eine Tendenz, sich an die Stelle eines im Hintergrund steuernden Staatspräsidenten zu setzen, anstatt die Rolle eines Schiedsrichters zwischen den politischen Kräften einzunehmen. Anders als das Parteiverbotsverfahren nach Art. 21 Abs. 2 GG vor dem Bundesverfassungsgericht, das im Erfolgsfalle nur die Feststellung der Verfassungswidrigkeit einer Partei kennt ${ }^{64}$, enthält Art. 68 Abs. 4 in Verbindung mit Art. 69 Abs. 6 TV eine nähere Umschreibung möglicher Konsequenzen, ohne dem weiten Auslegungsspielraum des Gerichts Grenzen zu setzen. Art. 69 TV und seine Konkretisierung im Parteiengesetz eröffnen dem Gericht ein Spektrum an Sanktionen, die je nach der Schwere der vorliegenden Verstöße angeordnet werden können. ${ }^{65}$ Neben dem Verbot kommen die Verwarnung und die (teilweise) Versagung staatlicher Parteienfinanzierung in Betracht, wobei die Sanktionen in einem Stufenverhältnis zueinander stehen. ${ }^{66} \mathrm{Im}$ Vordergrund steht dabei die Bildung eines Brennpunktes, die in Art. 69 Abs. 6 S. 2 TV definiert ist. Eine politische Partei wird zum Brennpunkt verfassungswidriger Aktivitäten, wenn Parteimitglieder im großen Umfang Taten begehen, die Schutzgüter des Art. 68 Abs. 4 TV verletzen und führenden Parteifunktionären bekannt sind. Wie auch im Falle der Tugendpartei - Fazilet Partisi -, die in den 1990er Jahren verboten wurde und Vorläufer der AKP war, lag der Vorwurf im Verfahren gegen die AKP im Verstoß gegen das Laizismusprinzip. Im Ergebnis wurde die AKP „nur“, wenn auch mit knapper Mehrheit im Richterkollegium, zur „symbolischen“ Rückerstattung der Hälfte der staatlichen Zuschüsse für das laufende Jahr verurteilt. ${ }^{67}$ Das ist zwar kein „Freispruch“, jedoch auch kein - nunmehr verfassungsgerichtlich inszenierter - Putsch. Ob das Gericht bei seiner Entscheidung den Pfad zulässiger und nachvollziehbarer Auslegung gegangen ist, kann erst im Rahmen einer hier nicht zu leistenden, genauen Analyse der Urteilsbegründung evaluiert werden. Schon jetzt zeigt sich aber, dass die politischen Parteien keinen Zweifel mehr daran haben, dass die Verfassungsgerichtsbarkeit ein notwendiger Bestandteil eines demokratischen Verfassungsstaates ist.

\section{Paradigmenwechsel im türkischen Verfassungsleben?}

Mag man auch die Entscheidung des Verfassungsgerichts zur Wahl des Staatspräsidenten wegen ihrer weitreichenden Auslegung des Verfassungstextes kritisieren, so bleibt der Umstand bemerkenswert, dass in der konkreten politischen Krise das Verfassungsgericht zur Entscheidung angerufen wurde. Trotz verschiedentlich geäußerter Befürchtungen ${ }^{68}$, das Militär werde „eingreifen“, kam es dazu nicht. Im erneuten Anlauf zur Wahl des Staatsprä-

64 Katrin Stein, in: Jörn Ipsen (Hrsg.), Kommentar zum ParteienG, München 2008, Vor $\$ \$ 32,33$, Rn. $2 \mathrm{f}$.

65 Vgl. hierzu näher Christian Rumpf, Einführung in das türkische Recht, München 2004, $\$ 8$ Rn. 46.

66 Dies ergibt sich aus dem Wortlaut des Verfassungstextes. Art. 69 Abs. 6 S. 1 TV sieht vor, dass eine Entscheidung auf endgültige Schließung nur erfolgt, wenn die verfassungswidrige Betätigung zu einem Brennpunkt der parteilichen Aktivitäten geworden ist.

67 Vgl. dazu Dirk Tröndle, a.a.O. (Fn. 2), S. 69.

68 Vgl. auch die öffentliche Äußerung des Generalsekretärs des Europarates Terry Davis: Turkish military should stay in their barracks and keep out of politics, Pressemeldung des Europarates vom 29. April 2007, Nr. 271, www.coe.int./press. 
sidenten am 28. August 2007 nach den Neuwahlen am 22. Juli 2007 gelang es der Regierungsfraktion der AKP, durch parlamentarische Absprachen mit der Fraktion der MHP die vom Verfassungsgericht verlangten Anwesenheitsquoren trotz erneutem Wahlboykott durch die CHP-Fraktion zu erfüllen und den erneut kandidierenden Abdullah Gül im dritten Wahlgang zu wählen. Die Verfassungsmäßigkeit dieser Wahl wurde bisher von keiner Seite angezweifelt. Die politischen Gegner haben sich, wie es scheint, mit dieser durch die vorangegangene Neuwahl des Parlaments aufs deutlichste demokratisch legitimierten Entscheidung abgefunden. Möglicherweise deutet sich damit ein Paradigmenwechsel im türkischen Verfassungsleben an. Auch wenn man die Begründung der dargestellten Entscheidung und auch die politischen Umstände ihres Zustandekommens für fragwürdig hält, muss man anerkennen, dass die politische Krise diesmal innerhalb des rechtlichen Rahmens der Verfassung gelöst wurde. Dieser Rahmen wurde nicht unter Berufung auf „übergeordnete Prinzipien" mit Mitteln der Gewalt zerbrochen. Die beteiligten Akteure haben in dieser Situation dem Verfassungsgericht die Rolle des „Hüters der Verfassung“ überlassen. ${ }^{69}$ Ungeachtet der Erbitterung ${ }^{70}$, die die hier besprochene Entscheidung zum Teil hervorgerufen hat, kann dies als zukunftsweisende Perspektive für das Verfassungsgericht gelten. Die eher symbolische Verurteilung der AKP im Parteiverbotsverfahren 2008 könnte ein weiteres Indiz dafür sein, dass das Gericht seine Rolle im modernen Sinne neu definiert.

War eine Demokratisierung der Türkei bereits mit den Verfassungsänderungen der Jahre 2001 und $2004 \mathrm{zu}$ erkennen, bleibt aus rechtsstaatlicher Sicht festzuhalten, dass mit der Anrufung des Verfassungsgerichts im Fall der Präsidentenwahl die politischen Akteure einen brisanten Konflikt in den Bahnen des Verfassungsrechts gelöst und dadurch zur Rechtssicherheit und -klarheit in ihrem Land beigetragen haben.

69 In diese Richtung auch Mithat Sancar, Staatsrechtslehrer und Publizist, in einem Interview mit dem Neuen Deutschland vom 28. August 2007, http://www.nd-online.de/artikel.asp? $\mathrm{AID}=115238 \& \mathrm{IDC}=2$ (Abruf am 31. August 2007).

70 So bezeichnete Ministerpräsident Erdoğan (AKP) das Urteil des Verfassungsgerichts laut eines Berichts im Focus vom 2. Mai als „Schlag ins Gesicht der Demokratie“, http://www.focus.de/ politik/ausland/tuerkei_aid_55102.html (Abruf am 1. September 2007). 